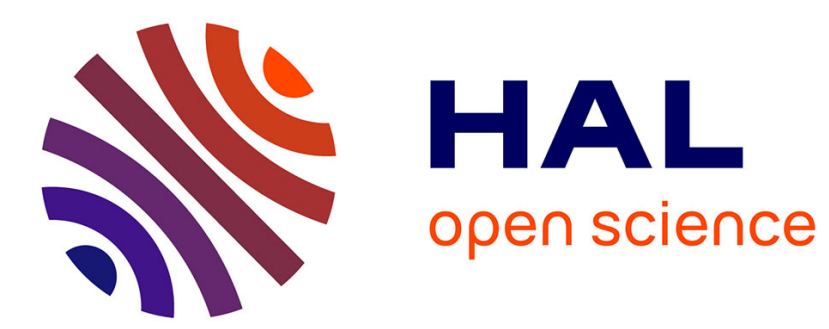

\title{
Oxydation et protection des alliages base niobium
}

\author{
Jean Steinmetz, Michel Vilasi, Bernard P. Roques
}

\section{To cite this version:}

Jean Steinmetz, Michel Vilasi, Bernard P. Roques. Oxydation et protection des alliages base niobium. Journal de Physique IV Proceedings, 1993, 03 (C9), pp.C9-487-C9-498. 10.1051/jp4:1993952 . jpa00252392

\section{HAL Id: jpa-00252392 https://hal.science/jpa-00252392}

Submitted on 1 Jan 1993

HAL is a multi-disciplinary open access archive for the deposit and dissemination of scientific research documents, whether they are published or not. The documents may come from teaching and research institutions in France or abroad, or from public or private research centers.
L'archive ouverte pluridisciplinaire HAL, est destinée au dépôt et à la diffusion de documents scientifiques de niveau recherche, publiés ou non, émanant des établissements d'enseignement et de recherche français ou étrangers, des laboratoires publics ou privés. 


\title{
Oxydation et protection des alliages base niobium (*)
}

\author{
Jean Steinmetz, Michel Vilasi et Bernard Roques
}

Laboratoire de Chimie du Solide Minéral, U.A. CNRS n ${ }^{\circ}$ 158, Université de Nancy I, B.P. 239, 54506 Vandoeuvre les Nancy Cedex, France

\begin{abstract}
The ceaseless demand for higher temperatures in aerospace systems explains the research programs about both the intermetallic compounds and the refractory metals. Concerning refractory metals, niobium has received the greatest attention, because of its good physical and mechanical properties (melting point, density, ductile-brittle transition temperature, strength above $1000^{\circ} \mathrm{C}$ ). Nevertheless, the bad oxidation behaviour of niobium or niobium alloys has excluded their use whithout protective coatings, as important research from 1955 to 1975 has clearly proved it. Despite this severe handicap, an intensive research is again conducted since some years, on niobium alloys, bare or coated. These recent investigations profit by new techniques in domains of alloys elaboration or coatings deposition. This paper also will treat our niobium program, which is focused in two main directions : developments of new niobium alloys structures, copied from $\gamma / \gamma^{\prime}$ superalloys structure, and protective coatings elaborated by pack cementation.
\end{abstract}

\section{Introduction.}

Après 40 ans de recherches plus ou moins intensives sur l'utilisation des métaux réfractaires et de leurs alliages dans des applications à haute température, la période actuelle connaît un nouveau et large regain d'intérêt pour des matériaux offrant une résistance mécanique à chaud élevée, notamment dans des secteurs touchant à la recherche spatiale et aéronautique [1].

Parmi les alliages réfractaires, ceux de niobium restent considérés comme les plus prometteurs pour des applications dynamiques (disques, aubes de turbines), comme pour d'autres, statiques (boucliers thermiques, systèmes d'injection, boulonnerie...). Ils présentent en effet plusieurs avantages par rapport à ceux de molybdène : une densité inférieure de 15 à $20 \%$, une température de transition ductile-fragile nettement plus basse et une plus grande facilité de transformation. Comparés à quelques alliages classiques, base nickel ou cobalt, ils leur opposent des valeurs de limites d'élasticité et de résistances à la rupture supérieures à températures élevées [2]. Enfin, l'apport récent de la métallurgie des poudres, grâce entre autres aux procédés de solidification rapide, de mécanosynthèse (mechanical alloying) et de densification (filage à chaud, pressage isostatique à chaud), permet d'améliorer les performances mécaniques de ces mêmes alliages, de pallier les difficultés de mise en forme d'autres, tel le WC-3009 (Nb - 30W - 9Hf), d'envisager aussi l'élaboration de nouveaux, qui associeraient des métaux à points de fusion très différents [2-6]. Par contre, ces alliages ont tous l'inconvénient d'une grande réactivité chimique ; de la même façon que le métal pur, ils s'oxydent rapidement dès 500 à $600^{\circ} \mathrm{C}$. Malgré l'évidence qu'une amélioration de leurs

(*) Keynote lecture. 
propriétés chimiques paraît difficilement conciliable avec le maintien de leurs performances mécaniques, une recherche intensive a repris en 1986 pour la mise au point d'alliages de niobium resistant à l'oxydation $[7,8]$. Conscients de la difficulté de la tâche, les chercheurs ont également exploré le domaine des revêtements protecteurs pour niobium et alliages de niobium, en tentant d'améliorer les solutions mises au point il y a 25 ans ou en orientant leurs travaux dans des voies plus originales. Le présent papier s'efforcera donc de présenter les derniers travaux menés sur ces sujets, après avoir rapidement rappelé ceux des acteurs du "premier round niobium", travaux qui ont conduit à l'élaboration d'alliages ou de revêtements incontournables et qui ont déjà fait l'objet de larges analyses [9, 10].

\section{Oxydation du niobium et des alliages.}

Toutes les études consacrées aux réactions du niobium avec l'oxygène ont montré que la cinétique de ces réactions était particulièrement complexe, en raison notamment de la formation de plusieurs types d'oxyde suivant la température et de la dissolution simultanée de l'oxygène dans le métal [11, 12]. Par ailleurs, la vitesse d'oxydation du niobium est trop importante quelle que soit la température. L'amélioration du "caractère protecteur" de l'oxyde $\mathrm{Nb}_{2} \mathrm{O}_{5}$, dont la croissance n'est pas indépendante de la pression d'oxygène [13], réside donc dans l'utilisation de l'une ou plusieurs des solutions suivantes :

- addition dans la couche d'oxyde de cations plus petits dans le but de réduire le rapport de Pilling et Bedworth (2,67 pour $\mathrm{Nb}_{2} \mathrm{O}_{5} / \mathrm{Nb}$ à comparer à 1,28 pour le couple $\left.\mathrm{Al}_{2} \mathrm{O}_{3} / \mathrm{Al}\right)$;

- addition de cations de plus basse valence afin d'augmenter la concentration de défauts et de ce fait la plasticité de l'oxyde ;

- stabilisation de $\mathrm{NbO}$ et $\mathrm{NbO}_{2}$ pour abaisser les contraintes ;

- addition de cations de valence supérieure $\left(\mathrm{W}^{\mathrm{VI}}, \mathrm{Mo}^{\mathrm{VI}}, \mathrm{Re}^{\mathrm{VI}}\right)$ qui réduiront l'épaisseur de la couche compacte de $\mathrm{Nb}_{2} \mathrm{O}_{5}$ dont la croissance obéirait à la loi de Wagner Hauffe.

L'effet de l'ajout de quelques éléments métalliques au niobium est illustré par la figure 1 qui montre que la réduction de la vitesse d'oxydation reste limitée dans les cas les plus favorables. La plupart des éléments d'alliages à des teneurs inférieures à $10 \%$ réduisent l'oxydation, mais pour des teneurs supérieures, certains éléments comme le chrome et plus encore le vanadium accélèrent le processus. Les meilleures compositions ont été obtenues avec des alliages conduisant à la stabilisation de $\mathrm{NbO}$ sous $\mathrm{NbO}_{2}$ et $\mathrm{Nb}_{2} \mathrm{O}_{5}$, dans le cas du système $\mathrm{Nb}-\mathrm{Al}-\mathrm{V}$ [15], ou à la formation d'oxydes mixtes, de structure rutile préférentiellement, par l'ajout de $\mathrm{Ti}$ [16], $\mathrm{Ti}+\mathrm{W}$ [17], $\mathrm{Al}+\mathrm{M}$ où $\mathrm{M}=\mathrm{Cr}, \mathrm{Fe}, \mathrm{Co}, \mathrm{Ni}$ [18]. Toutefois, le plus souvent, ce gain de résistance à l'oxydation se fait au détriment de la résistance mécanique.

Malgré ce bilan peu encourageant la société Lockheed Missiles \& Spaces a mis sur pied en 1986, en collaboration avec l'Université de Pittsburgh, un important programme de recherche consacré à l'évaluation des possibilités de développer des couches protectrices d'alumine sur alliages de niobium [17]. Le projet est ambitieux quand on songe que dans le système binaire $\mathrm{Nb}-\mathrm{Al}$, l'aluminiure $\mathrm{NbAl}_{3}$ n'est protégé que temporairement par $\mathrm{Al}_{2} \mathrm{O}_{3}$ $[19,20]$. Même si l'addition de chrome et yttrium favorise la protection sur des durées plus longues, l'aluminiure reste handicapé par son manque de ductilité, sa faible ténacité et sa difficulté de mise en œuvre $[21,22]$. Pour tenter d'abaisser la concentration critique d'aluminium nécessaire à la formation d'une couche compacte d'alumine, Perkins et al. [7, $8,23,24]$ ont exploité le modèle de Wagner [25] sur l'oxydation sélective d'un élément dans 


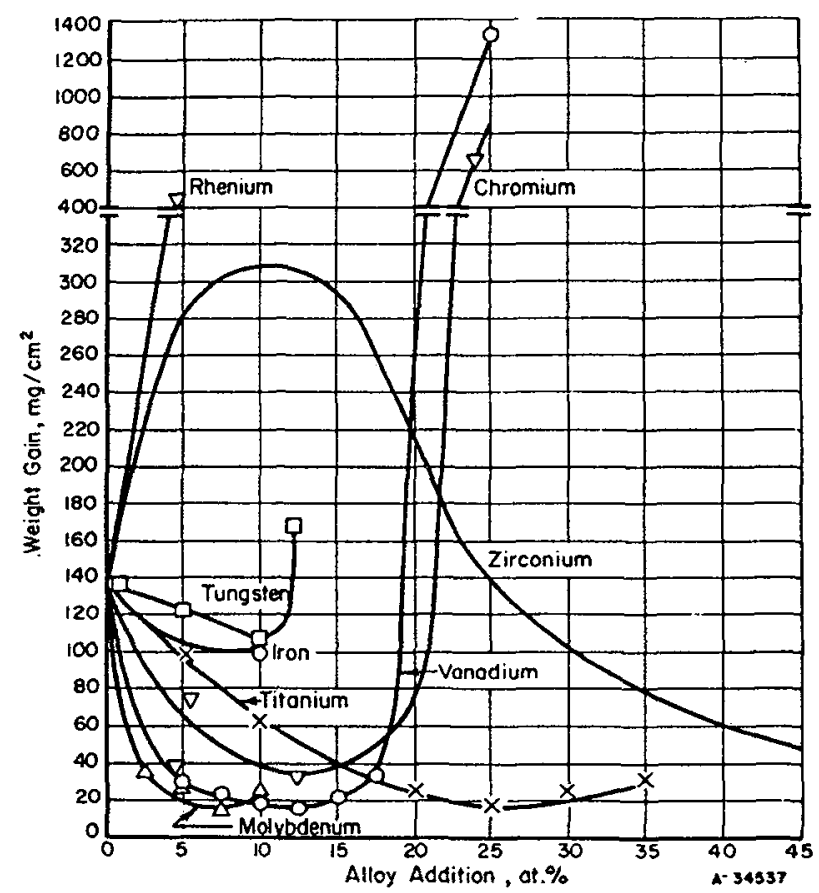

Fig. 1. - Gains de poids d'alliages de niobium exposés 5 heures dans l'air sec à $1000^{\circ} \mathrm{C}$ [14].

un alliage :

$$
N_{\mathrm{Al}(\text { crit. })}=\left[\frac{\pi}{3} g^{*} N_{\mathrm{O}} \frac{D_{\mathrm{O}} V_{\mathrm{M}}}{D_{\mathrm{Al}} V_{\mathrm{Ox}}}\right]^{1 / 2}
$$

où $N_{\mathrm{O}}=$ solubilité de l'oxygène dans l'alliage ; $D_{\mathrm{O}}, D_{\mathrm{Al}}=$ diffusivités de l'oxygène et de l'aluminium dans l'alliage ; $V_{\mathrm{M}}$ et $V_{\mathrm{Ox}}=$ volumes molaires de l'alliage et de l'oxyde ; $g^{*}=$ fraction volumique critique d'oxydes.

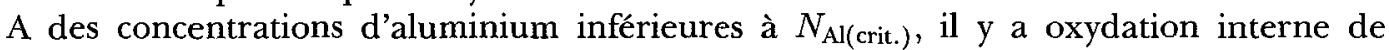
l'aluminium et les trois paramètres qu'il est important de contrôler sont donc $N_{\mathrm{O}}, D_{\mathrm{O}}$ et $D_{\mathrm{Al}} . N_{\mathrm{O}}$ peut être réduit par addition d'éléments ayant un rapport électrons par atome $(e / a)$ plus élevé que $\mathrm{Nb}(\mathrm{Cr}, \mathrm{Mo}, \mathrm{W}, \mathrm{Mn}, \mathrm{Ru}, \mathrm{Re})$ [26]. La diffusivité de l'oxygène sera abaissée en ajoutant des éléments tels $\mathrm{Zr}$ ou $\mathrm{Hf}$, plus oxydables que $\mathrm{Nb}$, ou d'autres possédant un rayon atomique plus petit comme $\mathrm{Cr}, \mathrm{V}, \mathrm{Mn}$, tandis que Ti et $\mathrm{V}$ augmenteront la solubilité de $\mathrm{Al}$ dans une structure de type $\mathrm{A}_{2}$. C'est ainsi que les alliages de composition voisine de $\mathrm{NbTiAl}_{2}$ et modifiés par 3 à 5 at $\%$ de $\mathrm{Gr}$ et $\mathrm{V}$ deviennent alumine formeurs au-dessus de $1350{ }^{\circ} \mathrm{C}$, $N_{\mathrm{Al}}$ pouvant même approcher 37 at\%. A plus basse température, l'alliage de structure $\mathrm{B}_{2}$, ou $\mathrm{A}_{2}$ ordonnée, se transforme en une structure fine mais biphasée composée de 2 aluminiures, TiAl $\gamma$ et $\mathrm{Nb}_{2} \mathrm{Al} \sigma$ [27], et perd son caractère alumine formeur. D'autre part, l'addition de $10 \%$ de silicium à un alliage $\mathrm{Nb}-\mathrm{Ti}-\mathrm{Al}$ contenant 40 à 45 at\% de $\mathrm{Al}$ le rend alumine formeur à $1100^{\circ} \mathrm{C}$ dans l'air, mais cette teneur élevée en silicium fragilise l'alliage tout en abaissant de 40 à $50^{\circ} \mathrm{C}$ son point de fusion [24]. A la suite de ce travail Perkins et al. furent amenés à conclure que la réalisation d'alliages de niobium ductiles et combinant à chaud de bonnes 
propriétés mécaniques avec une résistance à l'oxydation convenable restait problématique. Cependant certains alliages du type " $\mathrm{NbTiAl}_{2}$ " pourraient être utilisés comme matrices de composites intermétalliques ou comme revêtements de substrats plus oxydables.

Il reste toutefois la possibilité de développer des alliages à dispersion d'oxydes, de carbures, de nitrures ou d'intermétalliques, en utilisant de préférence la voie de la métallurgie des poudres, pour une meilleure distribution de phases plus fines et une ductilité supérieure [2]. Nous donnerons en exemple la réalisation par "mechanical alloying" d'un matériau composite associant une matrice de l'alliage B- $88\left(\mathrm{Nb}-28 \mathrm{~W}-2 \mathrm{Hf}\right.$ ) à un mélange $\mathrm{NbAl}_{3} / \mathrm{NbFe}_{2}$ et dont la vitesse d'oxydation serait plus faible que celle d'un alliage coulé $\mathrm{Nb}-\mathrm{Fe}-\mathrm{Al}$ [28]. Ce résultat, diversement apprécié $[2,24]$, est un de ceux qui ont conduit Loria à proposer pour l'obtention de résistances mécaniques et chimiques élevées à chaud l'introduction dans un composé si possible alumine formeur d'une seconde phase qui bloquerait le mouvement des dislocations [2]. Cette proposition précédait les travaux de Perkins et al. mais elle reste intéressante si la phase intermétallique en dispersion peut assurer un transport rapide d'aluminium vers la surface. Une telle structure, calquée sur celle des superalliages de type $\gamma-\gamma^{\prime}$ a été proposée récemment par Naka [29]. Elle peut être obtenue par la précipitation, dans une matrice base niobium $A_{2}$, d'un intermétallique de type $B_{2}$ ou $L 2_{1}$. Comme parmi les quelques 300 binaires $B_{2}$ recensés aucun ne contient du niobium, il convient de rechercher la solution dans des systèmes ternaires ou plus complexes, qui combinent $\mathrm{Nb}$ et $\mathrm{Al}$ à un ou plusieurs autres éléments. Un deuxième impératif est la mise en équilibre de deux phases ayant des paramètres de maille aussi proches que possible $\left(a_{\mathrm{Nb}}=3,30 \AA\right)$. Le troisième n'est pas minime si on considère qu'il faut trouver des conditions telles que l'aluminiure, pas trop fragile de préférence, précipite dans une matrice de niobium ductile. Dans le cas contraire, la mécanosynthèse peut offrir une solution plus souple que celle de la fusion.

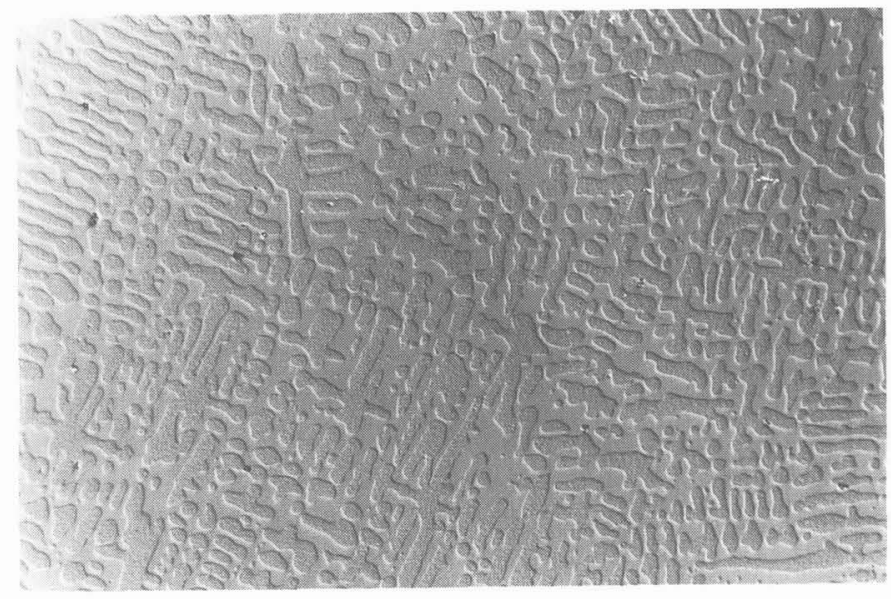

Fig. 2. - Alliage biphasé Nb-PdAl préparé par fusion inductive et recuit 8 jours à $1100^{\circ} \mathrm{C}(\times 437)$.

Pour notre part, nous avons mis en application ces idées sur une famille d'alliages biphasés choisie dans les systèmes Nb-Al-Pd(Ru). Trois facteurs nous ont conduits à examiner plus attentivement les systèmes $\mathrm{Nb}-\mathrm{Pd}-\mathrm{Al}$ et $\mathrm{Nb}-\mathrm{Ru}-\mathrm{Al}$ :

- l'existence de phases de type $\mathrm{B}_{2}$ dans les aluminiures de métaux précieux ; 
- l'influence positive de ces mêmes métaux sur l'oxydation de superalliages base nickel $[30]$;

- Ies résultats de Fleisher sur les composés potentiellement intéressants pour des applications à températures élevées [31].

Dans les deux cas des structures biphasées ont pu être obtenues (Fig. 2). Il reste que ces alliages sont fragiles (échelle 2 dans le "hammer test"), mais intéressants du point de vue de leur résistance à l'oxydation. A titre d'exemple, la figure 3 représente la section polie d'un alliage $\mathrm{Nb}_{2} \mathrm{PdAl}$ oxydé à $1100^{\circ} \mathrm{C}$, et recouvert d'une couche duplex $\mathrm{Al}_{2} \mathrm{O}_{3} / \mathrm{NbAlO}_{4}$, tandis que la figure 4 permet de situer les possibilités d'un alliage NbRuAl, modifié par Mo et $\mathrm{Cr}$, par rapport à quelques alliages de niobium.
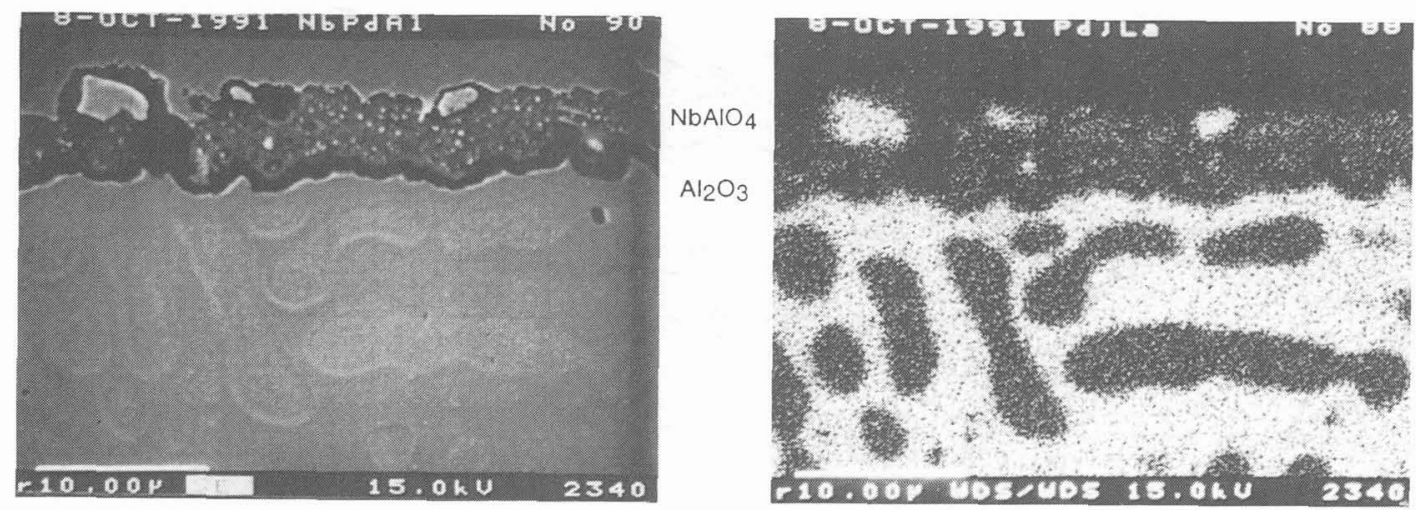

Fig. 3. - Alliage $\mathrm{Nb}_{2} \mathrm{PdAl}$ oxydé à $1100^{\circ} \mathrm{C}$ pendant 1 heure ; images électronique et $\mathrm{X}(\mathrm{Pd})$.

\section{Revêtements protecteurs pour alliages de niobium.}

En attendant la mise au point d'alliages de niobium à hautes caractéristiques mécaniques à chaud et possèdant une bonne résistance intrinsèque à l'oxydation, il faut se résigner à protéger les alliages classiques, comme le C-103 ou le Cb-752 (Nb-10W-2,5Zr) [32].

Les protections pour alliages de niobium restent essentiellement des revêtements à base de composés intermétalliques, siliciures ou aluminiures, à tel point que le revêtement mis au point par Priceman et Sama en 1965 constitue toujours le standard industriel [33]. Lélaboration de ce revêtement, proposé à 2 compositions, R512E à base de siliciures de chrome et fer et R512A à base de siliciures de chrome et titane, relève d'un procédé de dépôt de bouillies ou slurry. Les pièces en alliage de niobium sont trempées dans une bouillie contenant en dispersion des poudres de $\mathrm{Fe}, \mathrm{Cr}$ et $\mathrm{Si}$ dans les proportions en poids 20-20-60, proches d'un point eutectique à $1370^{\circ} \mathrm{C}$. Des traitements thermiques ultérieurs provoquent l'élimination du liant, la formation de siliciures complexes par réaction avec le métal de base et la consolidation enfin du revêtement dans une étape ultime de diffusion ( $1 \mathrm{~h}$ sous $10^{-3}$ torr à $1400^{\circ} \mathrm{G}$ ). Lépaisseur du revêtement peut atteindre $150 \mu \mathrm{m}$ et, quel que soit le substrat $(\mathrm{Nb}$ pur, Cb-752, WC-3009, Nb-46, 5Ti, D-43 (Nb-10W-1Zr)), il se compose de plusieurs siliciures dont les teneurs en métalloïde varient de $37,5 \mathrm{at} \%\left(\mathrm{Nb}_{5} \mathrm{Si}_{3}\right)$ côté substrat à $66,7 \%$ dans $\mathrm{NbSi}_{2}$ 


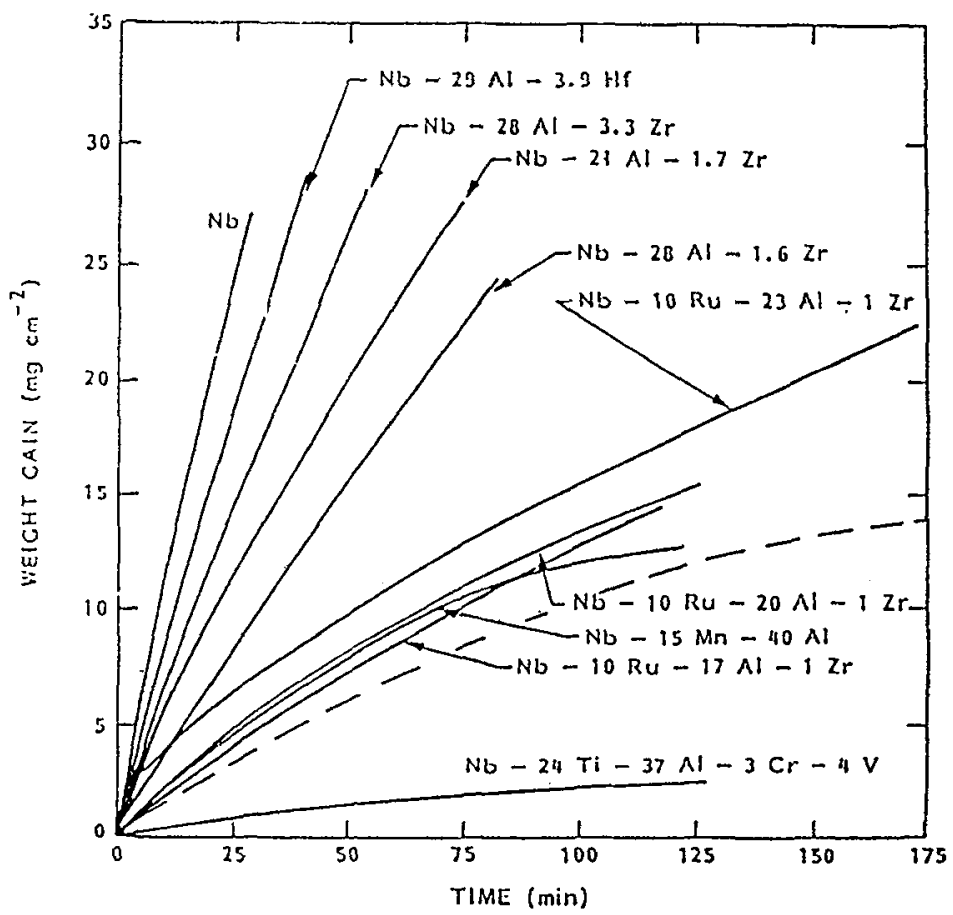

Fig. 4. - Oxydation à $1100^{\circ} \mathrm{C}$ d'alliages de niobium (sous $\mathrm{O}_{2}$ ), d'après [23] et de l'alliage $\mathrm{Nb}(\mathrm{Mo}, \mathrm{Cr}$ ) RuAl biphasé $\mathrm{A}_{2}-\mathrm{L} 2_{1}$ dans l'air (courbe en pointillés).

externe $[33,34]$. Les concentrations en silicium des phases intermédiaires trahissent sans aucun doute des isotypies avec les composés des familles structurales $\mathbf{M}_{11} \mathrm{Si}_{8}, \mathrm{M}_{6} \mathrm{Si}_{5}$ et $\mathrm{M}_{7} \mathrm{Si}_{6}$ où $M$ peut représenter un, deux ou trois métaux de transition (35-37), (Fig. 5). Nous citerons quelques exemples d'applications de ce revêtement sur :

- des boucliers thermiques de navette spatiale en $\mathrm{Cb}-752$;

- des orifices de guidage de navette ;

- des buses d'injection pour moteurs de fusées en alliage C-103 ou FS-85 (Nb-11W-10Ta) (moteurs RL 10 de Pratt et Whitney des fusées Atlas et Titan);

- des volets d'injection en alliage C-103 de la turbine Pratt et Whitney de l'avion F 100.

Lautre voie chimique importante d'élaboration de revêtements protecteurs est la cémentation activée, malgré les critiques la concernant :

- les difficultés de sa mise en auvre et son prix de revient élevé pour la protection de grosses pièces ;

- Les risques de contamination par les halogénures utilisés comme activants ;

- et surtout, l'inaptitude de ce procédé à former des revêtements fortement modifiés, au moins en une seule opération.

Nos études ont montré que ce dernier reproche était loin d'être fondé et que les risques de contamination ne sont pas très grands [38-40]. En un seul traitement de cémentation, il est possible d'obtenir des revêtements de siliciures alliés à chrome et fer et dans lesquels le niobium est un constituant minoritaire. La cémentation se déroule en régime de diffusion pure et ses résultats sont parfaitement reproductibles, la composition et la séquence des couches étant déterminées par le chemin de diffusion dans le couple constitué par le cément et l'alliage 


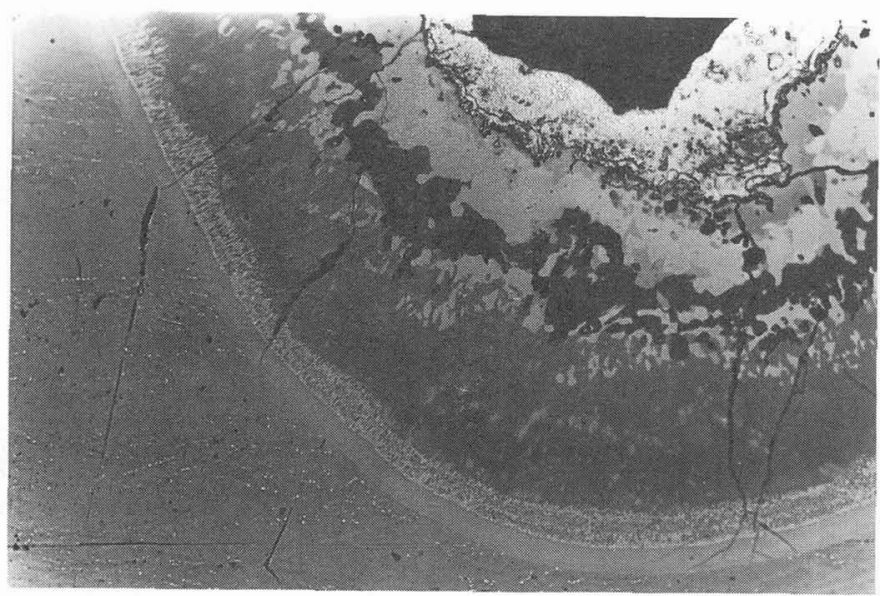

Fig. 5. - Revêtement R 512 E élaboré sur l'alliage P353M (Nb-10W-1,5V-3Zr-0,5Ti).

traité. Le revêtement $\mathrm{NbCrFeSi}$ obtenu à $1200^{\circ} \mathrm{C}$ en présence de $\mathrm{SiCl}_{4}$ s'apparente au dépôt R 512 E (Fig. 6). Les différentes phases ont été analysées à la microsonde électronique, synthétisées séparément puis cristallisées. Ce travail a conduit à une compréhension enfin claire des diagrammes d'équilibre $\mathrm{Nb}-\mathrm{Fe}-\mathrm{Si}$ et $\mathrm{Nb}-\mathrm{Cr}-\mathrm{Si}$ et à la caractérisation de phases ternaires et quaternaires totalement inédites [37, 41, 42], (Figs. 7 et 8). D'autre part l'état de surface des pièces cémentées est remarquable (Fig. 9) et son enrichissement superficiel en chrome en fait un revêtement $\mathrm{Cr}_{2} \mathrm{O}_{3}$ formeur.

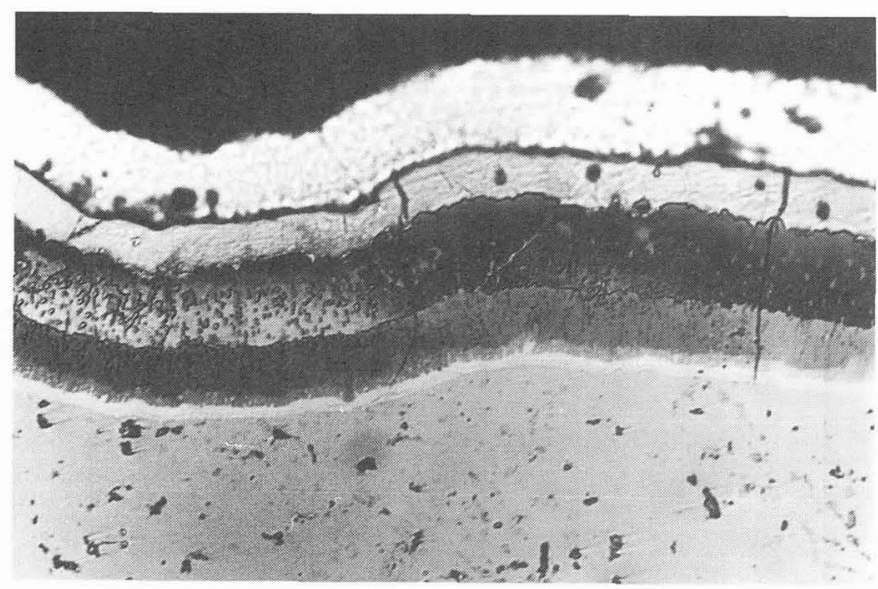

Fig. 6. - Revêtement, type pack cémentation, déposé à $1200^{\circ} \mathrm{C}$ sur l'alliage P353M.

Ces revêtements de siliciures sont tous fissurés, quel que soit le mode d'élaboration, par suite des différences importantes entre les coefficients de dilatation du substrat et des siliciures 


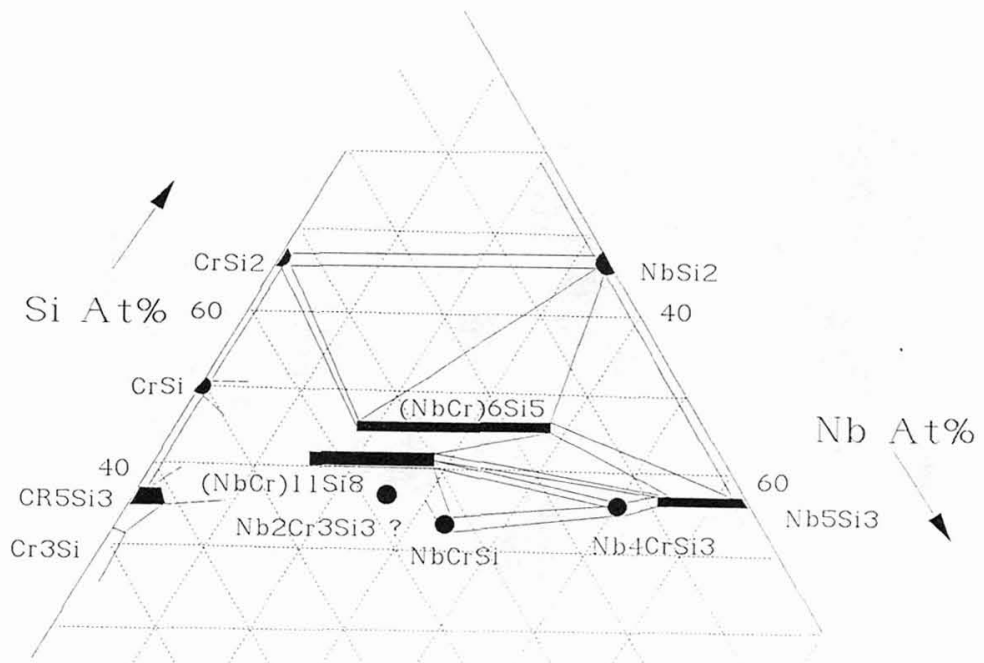

Fig. 7. - Diagramme d'équilibre Nb-Cr-Si à $1200^{\circ} \mathrm{C}$.

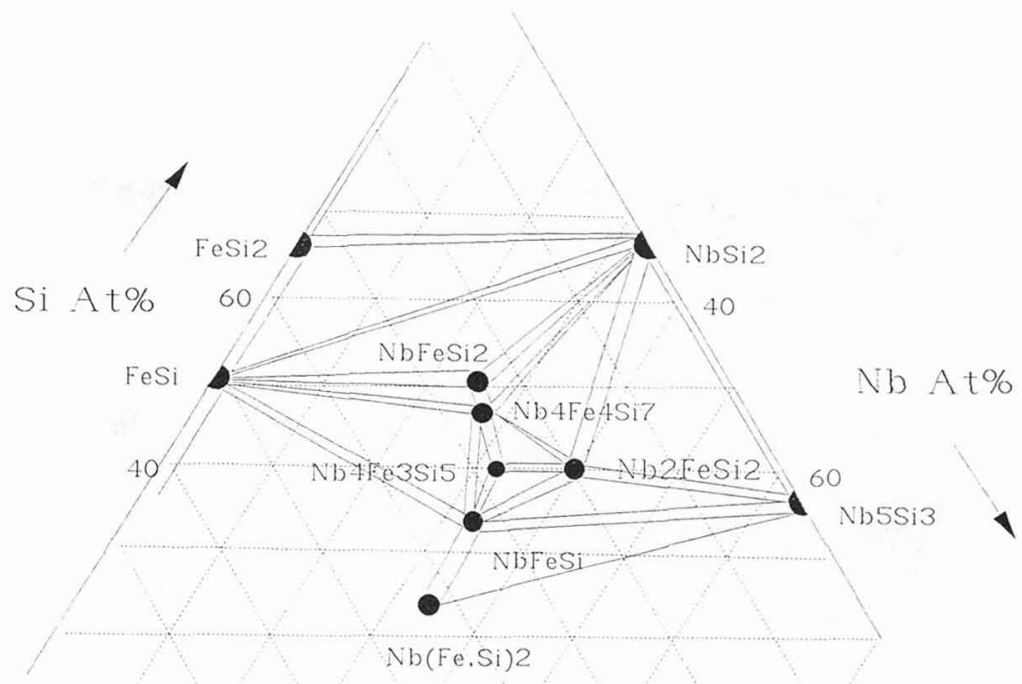

Fig. 8. - Diagramme d'équilibre Nb-Fe-Si à $1200^{\circ} \mathrm{C}$. 

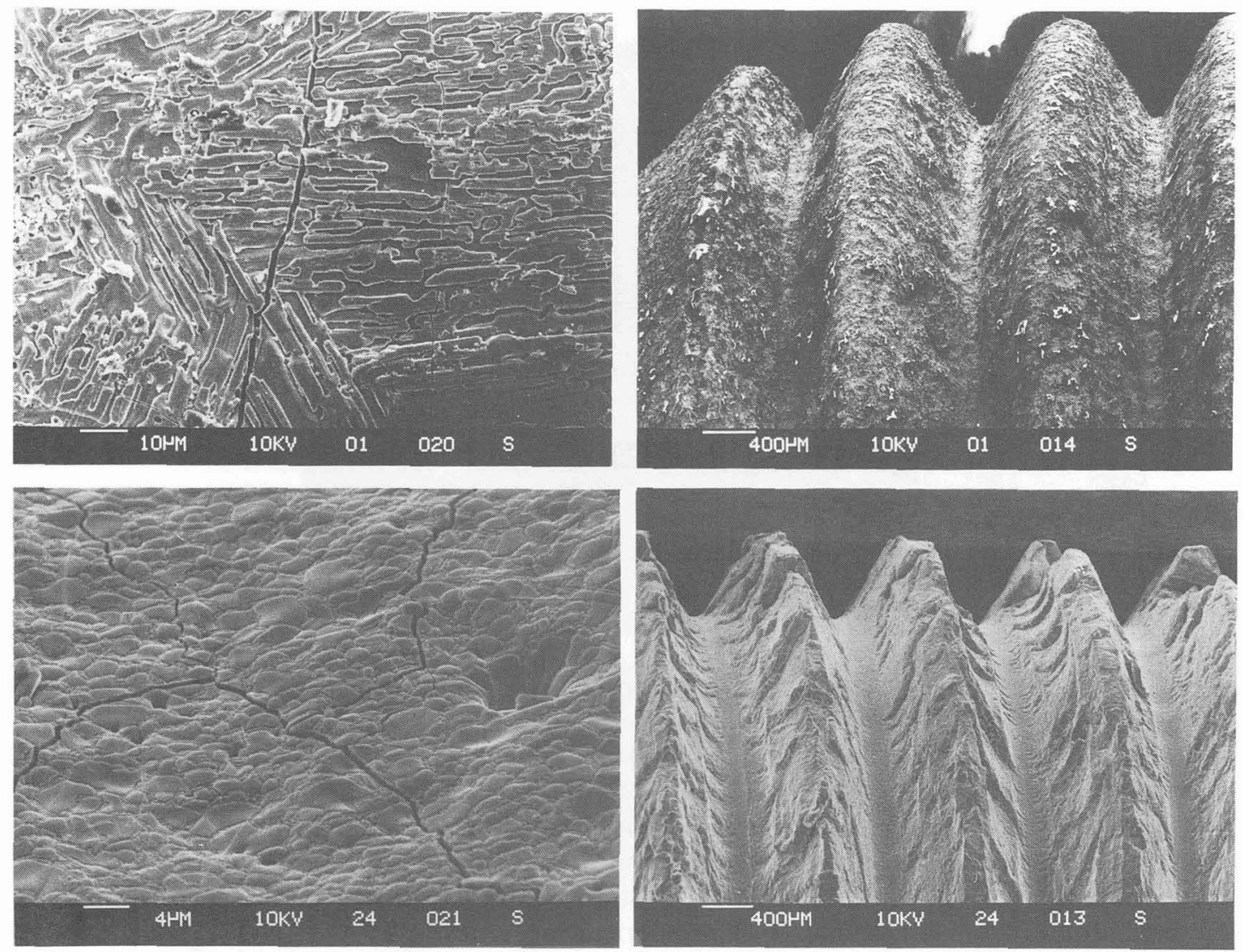

Fig. 9. - Etats de surface d'éprouvettes de traction en alliage P353M et revêtues par slurry ou par cémentation activée.

protecteurs (Figs. 5 et 6 ). Alors qu'à température élevée ces composés peuvent absorber un niveau de contraintes assez élevé par déformation plastique, à plus basse température ils deviennent fragiles et se trouvent en tension. L'émission acoustique a montré que le revêtement "slurry" se fissurait au refroidissement en-dessous de $1000^{\circ} \mathrm{C}$ et que la vitesse de formation des fissures atteignait un maximum dans la plage $800-1000^{\circ} \mathrm{C}$, selon le type de revêtement [24]. Ces fissures peuvent se cicatriser au cours d'un cycle ultérieur tandis que de nouvelles apparaîtront au refroidissement suivant. Certaines se remplissent d'oxydes et s'élargissent alors au cours des cycles, sans effet catastrophique tant qu'elles sont bloquées sur le siliciure $\mathrm{Nb}_{5} \mathrm{Si}_{3}[43,44]$, (Fig. 10). Lorsque les fissures atteignent le substrat, ce qui reste difficilement prévisible, elles provoquent une rapide dégradation des pièces. L'industrie a tenté de résoudre ce problème de fiabilité en l'examinant d'un point de vue statistique.

La protection du niobium par le disiliciure de molybdène peut partiellement régler le problème de l'écart entre les coefficients d'expansion thermique [45]; c'est ce qui explique le regain d'intérêt suscité par ce type de revêtement [45-47]. De plus $\mathrm{MoSi}_{2}$ est particulièrement réfractaire $\left(T_{\mathrm{F}}=2210^{\circ} \mathrm{C}\right)$ et possède une densité faible $(6,05)$, un module d' Young élevé à température ambiante $(379 \mathrm{GPa})$ ainsi qu'une remarquable résistance à l'oxydation et à la corrosion dans le domaine $1000-1700{ }^{\circ} \mathrm{C}$. Enfin la diffusion du silicium provenant de 


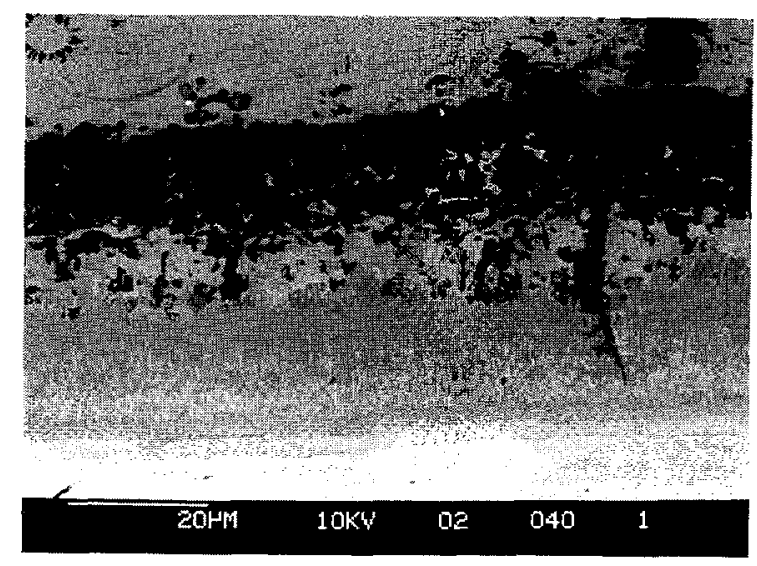

Fig. 10. - Fissures dans un revêtement type cémentation sur P353M après un essai de fluage de $1000 \mathrm{H}$ à $1050^{\circ} \mathrm{C}$ sous $150 \mathrm{MPa}$.

$\mathrm{MoSi}_{2}$ au travers de $\mathrm{Me}_{5} \mathrm{Si}_{3}(\mathrm{Me}=\mathrm{Nb}+\mathrm{Mo})$ est ralentie d'un facteur 3 à 4, par rapport à $\mathrm{Mo}_{5} \mathrm{Si}_{3}$ pur [45]. Cependant ce tableau est quelque peu terni par un phénomène de peste qui provoque, à basse température $\left(600^{\circ} \mathrm{G}\right)$, une oxydation catastrophique du siliciure [48]. L'addition de germanium qui diminue la différence de coefficient de dilatation entre $\mathrm{Si}(\mathrm{Ge}) \mathrm{O}_{2}$ et $\mathrm{MoSi}(\mathrm{Ge})_{2}$, permet cependant de limiter le phénomène [49]. Bianco et al. ont récemment exploité cette propriété pour élaborer sur niobium pur, par un procédé de pack cémentation (NaF), des revêtements $\mathrm{Mo}(\mathrm{Si}, \mathrm{Ge})_{2}$ ou $(\mathrm{Mo}, \mathrm{W})(\mathrm{Si}, \mathrm{Ge})_{2}$ dont les performances en oxydation cyclique à l'air à $1370^{\circ} \mathrm{C}$ sont encourageantes [47].

Les techniques de dépôt se sont diversifées au cours de ces dernières années avec l'apport des traitements physiques, laser et torche à plasma en autres [50,51]. Le deuxième procédé est utilisé par General Electric pour la projection d'un nouveau type de revêtement qui tranche avec les précédents. Il est appliqué sur des substrats également nouveaux, de type $\mathrm{Nb}(\mathrm{Hf}, \mathrm{Cr}) \mathrm{TiAl}(\mathrm{Al}<22$ at\%), ductiles, présentant une bonne résistance mécanique à chaud et pour certains plus résistants à l'oxydation que l'alliage commercial Cb-752. Leur composition doit correspondre à une structure $A_{2}$ (ou $B_{2}$ ) ou $A_{2} / N_{3} A l$ tandis que celle de leur revêtement, proche de la formule $\mathrm{Ru}_{14} \mathrm{Al}_{20} \mathrm{Cr}_{55} \mathrm{Fe}_{11}$, est celle d'un alliage biphasé $\mathrm{A}_{2}$ L2 1 . Ce système qui ne présenterait pas de perte de poids, même après 100 heures à $1500^{\circ} \mathrm{C}$, devrait être en équilibre thermodynamique avec un substrat de type $\mathrm{A}_{2}$, et suffisamment riche en aluminium pour que le revêtement n'évolue pas trop vite par diffusion.

\section{Conclusion.}

$\mathrm{Au}$ vu des documents publiés sur un sujet malgré tout protégé il faut reconnaître que peu de progrès vraiment décisifs paraissent avoir été réalisés depuis 25 ans sur la protection contre l'oxydation à chaud des alliages base niobium. L'apport de nouvelles techniques d'élaboration d'alliages ou de revêtements ainsi qu'une approche plus fondamentale des processus d'oxydation, notamment pour les alliages non revêtus, ne permettent pas encore d'envisager à court terme l'utilisation de niobium dans des pièces aussi sensibles que les disques ou aubes de turbomachines. Mais il reste que ces techniques ont incontestablement 
contribué à l'amélioration de la qualité des substrats et permis une plus grande souplesse dans le choix des compositions des dépôts protecteurs.

\section{Remerciements.}

Les auteurs remercient la Direction des Recherches Etudes et Techniques pour l'aide dont ils ont bénéficié au cours de ces dernières années sur le sujet de la protection des alliages de niobium.

\section{Bibliographie}

[1] Sims C.T., Adv. Mater. Processes 6 (1991) 32.

[2] LORIA E.A., J. Met. 7 (1987) 22

[3] Condliff A.F., J. Met. 9 (1986) 15.

[4] MUNSON M.C., J. Met. 5 (1989) 10.

[5] WoJcik C.C., High Temperature Niobium Alloys, J.J. Stephens, I. Ahmad Eds. (Warrendale, PA, 1989) p. 1.

[6] CERBA P., VILASI M., STEINMETZ J., contrat D.R.E.T., nº 89.34.179 (1992).

[7] PERKINS R.A., GHIANG K.T., MEIER G.H., AFOSR Contrat F 49620-86-C-0018, LMSC Report -F 195926 (February 1987).

[8] Perkins R.A., Chiang K.T., Meier G.H., Scr. Metall. 22 (1988). 419.

[9] STRINGer J., High Temperature Corrosion of Aerospace Alloys AGARD-AG-200 (NATO, August 1975).

[10] STRINGER J., High Temperature Corrosion of Aerospace Alloys AGARD-CP-120 (NATO, April 1972).

[11] JaHnke L.P., AIME Met. Soc. Conf., G.M. Ault, W.F. Barclay, H.P. Munger Eds. (Interscience New-York, 1963) p. 283.

[12] Catella G., Thèse Université de Nancy (1971).

[13] Sheasby J.S., Wallwork G.R., SMeltzer W.W., J. Electrochem. Soc. 113 (1966) 1255.

[14] Klopp W.D., MaYkuTH D.J., Sims C.T., JafFeE R.I., Battelle Memorial Institute Report BMI-1317 (1959).

[15] WLodek S.T., D.L. Douglass, Kunz Eds., AIME Met. Soc. Conf. (Interscience NewYork, 10, 1961) p. 553.

[16] FELten E.J., J. less-Common Met. 17 (1969) 185.

[17] Wlodek S.T., AIME Met. Soc. Conf., D.L. Douglass, Kunz Eds. (Interscience NewYork, 10, 1961) p. 175.

[18] Svedberg C., Properties of high Temperature Alloys, Z.A. Foroulis, F.S. Pettit Eds. (Pennington, N.J. : Electrochem. Soc., 1976) p. 331.

[19] RaIsSON G., Thèse Université de Nancy (1971).

[20] Grab Ke H.J.G., Steinhorst M., Brumm M., Wiemer D., Oxid. Met. 35 (3-4) (1991) 199.

[21] DoychaK J., Hebsur M.G., Oxid. Met. 36 (1-2) (1991) 113.

[22] Hebsur M.G., Oxid. Met. 36 (1-2) (1991) 171.

[23] Perkins R.A., Chiang K.T., Meier G.H., Miller R., Oxidation of High Temperature Intermetallics, T. Grobstein, J. Doychak Eds. (Warrendale, PA : TMS, 1988) p. 157. 
[24] Perkins R.A., Meier G.H., J. Met. 8 (1990) 17.

[25] WAGNER C., Z. Electrochem 63 (1959) 772.

[26] BRYANT R.T., J. Less-Common Met. 4 (1962) 62.

[27] JewetT T.J., Lin J.G., Bonda N.R., Seitzman L.E., Hsieh K.C., Chang Y.A., Perepezko J.H., Mater. Res. Soc. Symp. Proc. 133 (1989) 69.

[28] SVEDBERG R.G., AMMON R.L. WAESD-TR-84-0025 (June, 1984).

[29] NAKA S., KHAN T., JIMIS-6 Sendai (Japon, June 17-20, 1991).

[30] Costantini A., Thèse Université de Nancy (1990).

[31] Fleischer R.L., Zabala R.J., Metall. Trans. A 21 (1990) 2709.

[32] INNOYE H., Niobium, H. Stuart Ed. (TMS, 1984) p. 615

[33] PRiceman S., SAMA L., Electrochem. Tech. 6 (9-10) (1968) 31.

[34] JONES K.D., JAGKSON M.R., PEluso L.A., PRIBIS M.D., G.E. Tech. Inf. Ser. $\mathrm{n}^{\circ}$ 90CRD, $148(8)(1990)$.

[35] Steinmetz J., Malaman B., Roques B., J. Less. Common Met. 57 (1978) 133.

[36] STEInMETZ J., RoQUES B., J. Less-Common Met. 52 (1977) 247.

[37] Steinmetz J., Vilasi M., Venturini G., Malaman B., J. Alloys Met. Compounds 194 (1993) 127.

[38] Travaux de l'Université de Nancy, Contrat D.R.M.E., n ${ }^{\circ}$ 71.34241.00.480.75.01.

[39] Albrecht J.M., Aubry J., Roques B., Rev. Phys. Appl. Supplément au J. Phys. Colloque "Les journées Niobium" (Paris 20-21 oct, 1969) p. 551.

[40] RoQues B., Albrecht J.M., STEINMETZ J., Brevet Fr. 222455610 (1974).

[41] Steinmetz J., Roques B., Courtois A., Protas J., Acta Crystallogr. B 35 (1979) 2509.

[42] Vilasi M., Steinmetz P., Steinmetz J., J. Less-Common Met. 175 (1991) L7.

[43] BoRNSTEIN N.S., DE CRESCENTE M.A., US Patent 3764397, 10 (1973).

[44] PACker C.M., Oxidation of High-Temperature Intermetallics, T. Grobstein, J. Doychak Eds. (Warrendale, PA : TMS, 1988) p. 235.

[45] Fitzer E., SChlichting J., SCHMIdT F.K., High Temp.-High Press. 2 (1970) 553.

[46] Davydova A.D., Zotov Yu. P., IVAShchenko O.V., Kushnareva N.P., Yarosh I.P., Russ. Met. 1 (1990) 125.

[47] Bianco R., HARPer M.A., RAPP R.A., J. Met. 11 (1991) 20.

[48] Fitzer E., Plansee Proc. 2nd Seminar, Reutte/Tyrol (1955).

[49] Fitzer E., ReMmele W., 5th Int. Conf. on OComposite Materials, W.C. Harrigan, J. Strife, A.K. Dhingra Eds. (Met. Soc, 1985) p. 515.

[50] Pons M., Caillet M., Galerie A., Mater. Chem. Phys. 15 (1987) 423.

[51] JACKSON M.R., US Patent 331762 (march 1989), Dépôt en France nº 2645072 (5 oct. 1990). 